\title{
Context-Bounded Model Checking of LTL Properties for ANSI-C Software
}

\author{
Jeremy Morse, Lucas Cordeiro, \\ Bernd Fischer, Denis Nicole
}

\author{
Southampton \\ School of Electronics \\ and Computer Science
}

UFAM 


\section{Model Checking C}

Model checking:

- normally applied to formal state transition systems

- checks safety and temporal properties

Sofware model checking:

- models are abstractions, not necessarily precise

- no guarantee that model and software agree

BUT: $\mathrm{C}$ is difficult to model check:

- weakly typed $\Rightarrow$ conversion increase model complexity

- pointers $\Rightarrow$ indirections increase model complexity

- infinite state

- parts deliberately undefined, implementation- or host-specific

$\Rightarrow$ need to handle useful or common interpretations 
SMT-based bounded model checker for $\mathrm{C}$, based on CBMC:

- symbolically executes C into SSA, produces QF formulae

- unrolls loons un to a maximum bound

- assertior Goal: support LTL formulas in properties

- safety proporty overflows,...)

- user-specified properties

Multi-threaded programs:

- produces one SSA program for each possible thread interleaving

- interleaves only at "visible" instructions

- optional context bound 


\section{LTL - Linear Temporal Logic}

Supported operators:

- U: $p$ holds until $q$ holds

$p \cup q$

- $\mathrm{F}: p$ will hold eventually in the future

$\mathrm{F} p$

- G: $p$ always holds in the future

$\mathrm{G} p$

- $\mathrm{X}$ is not well defined for $\mathrm{C}$

- no notion of "next"

- C expressions used as atoms in LTL:

$\{$ keyInput $==1\} \rightarrow F \quad$ displaykeyup\}

(\{keyInput $!=0\} \mid\{$ intr $\}) \rightarrow$ G\{numInputs $>0\}$

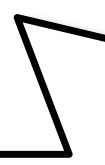

"event": change of global variable used in LTL formula 


\section{Büchi Automata (BA)}

- non-deterministic FSM over propositional expressions

- inputs infinite length traces

- acceptance $==$ trace passes through an accepting state infinitely often

- can convert from LTL to an equivalent BA

- use $1 \mathrm{t} 12 \mathrm{ba}$, modified to produce $\mathrm{C}$
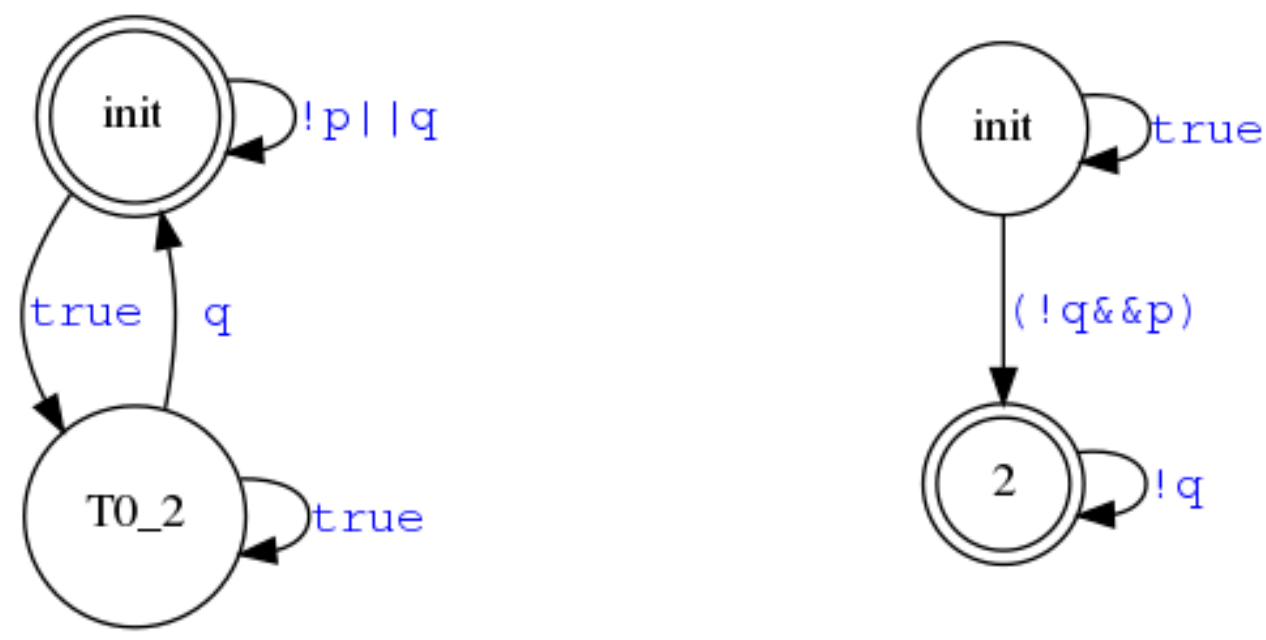

$p \rightarrow F q$

$!(p \rightarrow F q)$ 


\section{Using BAs to check the program}

- Theory: check product of model and never claim for accepting state

- SPIN: execute never claim in lockstep with model

- ESBMC:

- technically difficult to alternate between normal program and never claim program

- instead: run never claim program as a monitor thread concurrently with other program thread(s)

$\Rightarrow$ no distinction between monitor thread and other threads 


\section{Ensuring soundness of monitor thread}

Monitor thread will miss events:

- interleavings will exist where events are skipped (monitor thread scheduled out of sync)

$\Rightarrow$ can cause false violations of the property being verified

$\Rightarrow$ monitor thread must be run immediately after events

Solution:

- ESBMC maintains (global) current count of events

- monitor checks it processes events one at a time (using assume statements)

$\Rightarrow$ causes ESBMC to discard interleavings where monitor does not act on relevant state changes 


\section{Example monitor thread}

boo1 cexpr_0; // "pressed"

bool cexpr_1; // "charge > min"

typedef enum \{T0_init, accept_s2 \} 1t12ba_state;

1t12ba_state state $=$ T0_init;

unsigned int visited_states[2];

unsigned int trans_seen;

extern unsigned int trans_count;

State transition and "event" counter setup

void 1t12ba_fsm(bool state_stats) \{

unsigned int choice;

while(1) \{

choice = nondet_uint ();

$/ *$ Force a context switch */

yie1d();

atomic_begin();

assume (trans_count $<=$ trans_seen +1 );

trans_seen = trans_count;

个 only interleave whole block

reject unsafe interleavings 


\section{Example monitor thread}
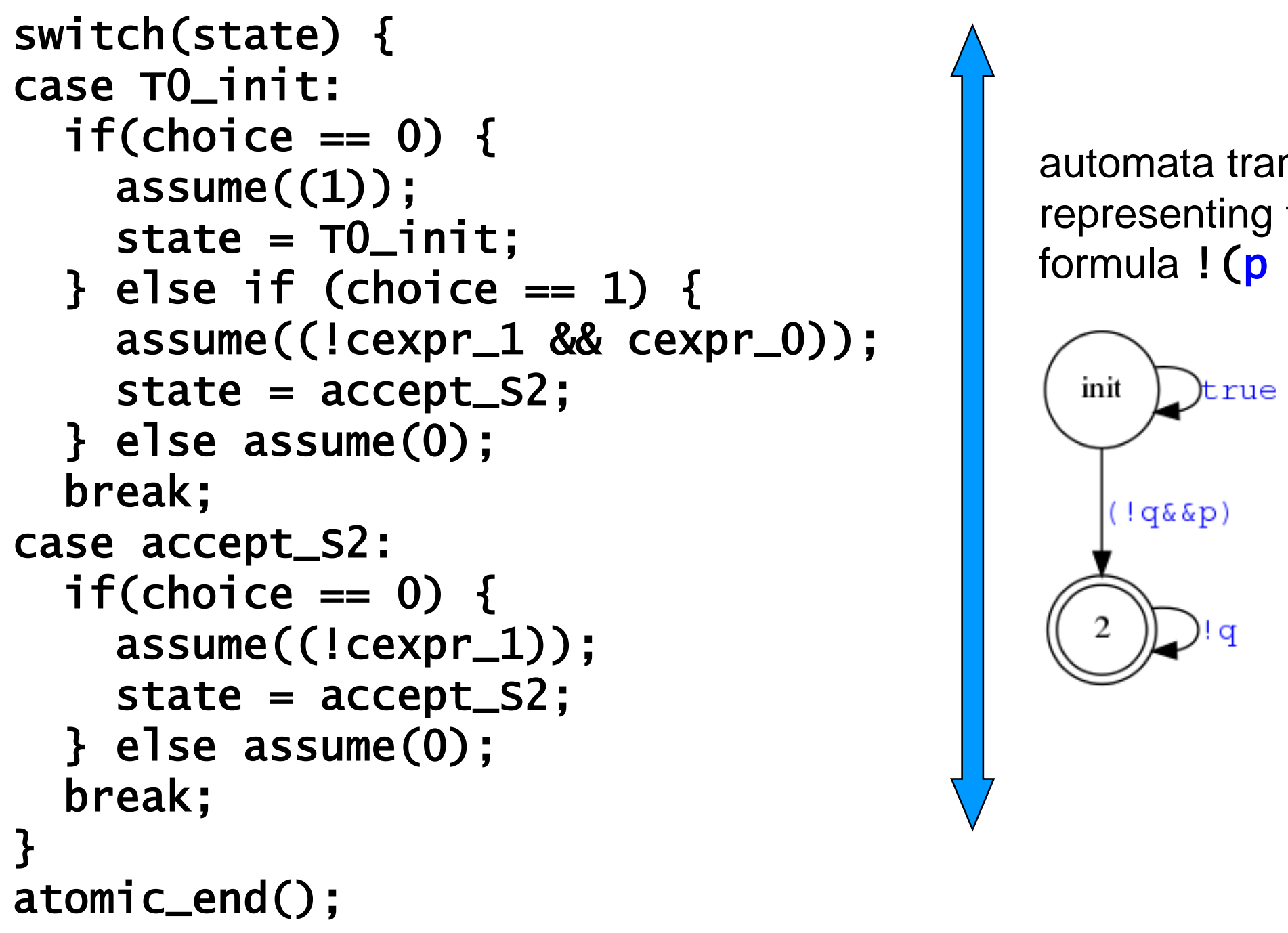


\section{Infinite traces and BMC?}

BMC forces program execution to eventually end

- but BA are defined over infinite traces...

Solution:

- follow SPINs stuttering acceptance approach: pretend final state extends infinitely

- re-run monitor thread after program termination, with enough loop iterations to pass through each state twice

- if an accepting state is visited at least twice while stuttering, BA accepts extended trace

- LTL property violation found 


\section{Experiments}

- checked properties of medical device firmware

- mostly of the form $p \rightarrow F q$ or (! $p \& \& F p) \rightarrow F q$

- tested against original code base, and code with seeded errors

- all properties shown to hold on original code, all seeded errors were found

\begin{tabular}{|c|c|c|}
\hline Test name & Interleavings & Elapsed time(s) \\
\hline start_btn & 7764 & 199 \\
\hline ub_btn & 3775 & 83 \\
\hline
\end{tabular}

approach requires large context switch bounds

serial_rx

5454

unwind bound:1, context bound: $\mathbf{4 0}$ 


\section{State Hashing}

- used to counter the state explosion problem in explicit-state model checking:

- variable assignments concatenated into state vector

- hash values used to record which states have been explored

- hash collisions prevent unique parts of the state space from being explored

- cannot be applied directly to symbolic model checking: variable assignments can contain non-deterministic values with constraints 


\section{Symbolic State Hashing}

Exploit SSA form:

- normalize RHS of each assignment in SSA form

- compute hash value and associate with LHS variable

- replace variable occurrences in RHS by variable hashes

- ... and re-hash

$\Rightarrow$ variables with same set of constraints hash to same values

$\Rightarrow$ independent of non-deterministic choices

- variable hashes and thread program counters concatenated into state vector

- rest as before...

- hash algorithm not important, we use SHA256 


\section{Symbolic State Hashing - Limitations}

- Equivalent states can have different hash values if:

- constraints are arranged in different orders

- (semantically) different sets of constraints

$\Rightarrow$ not all redundant states are removed

- However, we are primarily interested in reducing symmetry 


\section{State Hashing Experiments}

- same experiments, with state hashing enabled

- all tests decreased total runtime

- observable increase in amount of runtime per interleaving

\begin{tabular}{|c|c|c|c|c|}
\hline Test name & Interleavings & w / hashing & Elapsed time(s) & w / hashing \\
\hline start_btn & 7764 & 2245 & 199 & 71 \\
\hline up_btn & 3775 & 1385 & 83 & 37 \\
\hline keyb_start & 92795 & 49017 & 9796 & 4489 \\
\hline baud_conf & 485 & 419 & 17 & 16 \\
\hline serial_rx & 5454 & 3108 & 324 & 212 \\
\hline
\end{tabular}




\section{Relation to partial order reductions}

- Partial order reductions are the more common way to reduce number of redundant states explored

- demonstrably optimal method of doing this exists...

- ... but incurs additional complexity in detecting which context switches are redundant

- state hashing only eliminates the most obvious and immediate duplicate states...

- ... but only at the cost of extra overhead in symbolic execution

- detailed comparison remains future work 


\section{Conclusions}

- BMC framework can be extended to check ANSI-C software against an LTL formula (with reasonable efficiency)

- State hashing can be extended to symbolic model checking

- Runtime performance is improved by a modest amount by the use of state hashing

\section{Future Work}

- Full comparison of state hashing with POR

- Evaluate how effective such optimisations are when run on a distributed system 